\title{
Estudo da eficiência de empresas distribuidoras de jornais usando análise envoltória de dados
}

\author{
Luiz Cesar Nanci \\ Universidade Federal Fluminense \\ Programa de Mestrado em Engenharia de Produção \\ cnanci@predialnet.com.br \\ Sérgio Medeiros Azeredo \\ Universidade Federal Fluminense \\ Programa de Mestrado em Engenharia de Produção \\ smazeredo@uol.com.br
}

João Carlos C. B. Soares de Mello

Universidade Federal Fluminense

Departamento de Engenharia de Produção

jcsmello@producao.uff.br

\begin{abstract}
A dificuldade de se avaliar eficiência de operadores logísticos consiste no fato de geralmente se tratar de um problema de múltiplos inputs e outputs. A Análise Envoltória de Dados é um método não-paramétrico que elimina esta dificuldade e avalia unidades de produção de forma comparada. O objetivo deste trabalho é avaliar a eficiência das empresas distribuidoras de jornais usando a metodologia DEA - Análise Envoltória de Dados - especificamente na atividade de entrega domiciliar de jornais, na região metropolitana do Rio de Janeiro. Foi utilizado o modelo DEA clássico CRS. A principal dificuldade encontrada ao se avaliar esta atividade refletiu-se nas características geográficas e sócio-econômicas das áreas de atuação da cada empresa.
\end{abstract}

Palavras-chave: DEA; eficiência; distribuição domiciliar de jornais.

Assessing services of third-party logistics usually involve multiple inputs and outputs, what makes it a difficult task. Data Envelopment Analysis is a non-parametric method that eliminates this difficulty and assess production units comparing each other. The goal of this work is to assess third-party logistics, specifically in the activity of newspaper home delivery, using DEA classic CRS model. The main difficulty in assessing this activity is reflected in some geographic and social-economical characteristics of the areas of delivery.

Keywords: DEA; efficiency; newspaper home delivery.

\section{Introdução}

Em geral, o objetivo de medir a eficiência comparada entre unidades de produção é classificá-las em eficientes ou não-eficientes e dar uma medida relativa da eficiência para as não-eficientes. Outros objetivos da metodologia DEA consistem em estabelecer um ou mais benchmarks e posicionar as outras unidades em relação a eles ou ordená-las segundo as eficiências calculadas.

Na década de 90, as aplicações da metodologia DEA foram amplamente exploradas em diversos setores da economia, dentre eles o setor de Transportes e Logística, incluindo aplicações militares, serviços de ônibus escolares e avaliação de tráfego aéreo. Novaes
(2001) aplicou a metodologia DEA para avaliar a eficiência de operadores logísticos que operam no país e estabelecer benchmarks entre unidades regionais de um serviço de distribuição de produtos. Outros casos do uso de DEA no setor de Transportes podem ser encontrados em Soares de Mello et al. (2003), Angulo Meza et al. (2002) e Adler e Golany (2001).

A dificuldade de se avaliar operadores logísticos está no fato das atividades envolvidas possuírem muitas variáveis tanto de entrada (inputs) quanto de saída (outputs), o que estatisticamente só é tratável ao isolar-se um output de cada vez (variável dependente) e calcular-se os coeficientes de regressão para as variáveis independentes (inputs). Em DEA, é possível 
obter um índice único de eficiência, mesmo com múltiplos inputs e outputs.

\section{Estudo de caso}

A Infoglobo Comunicações LTDA é uma empresa das Organizações GLOBO, que edita e produz os jornais O GLOBO, um dos maiores e mais antigos do país em sua categoria, e mais recentemente o jornal EXTRA. A empresa produz ainda os jornais Valor Econômico e o Diário de São Paulo.

O jornal O GLOBO, foco deste estudo, circula em todas as capitais estaduais do Brasil, e sua produção se dá no Estado do Rio de Janeiro, onde sua distribuição é concentrada. Há ainda o serviço de entrega domiciliar, que disponibiliza o jornal diariamente ao cliente, em seu domicílio.

A operação de entrega domiciliar abrange toda a cidade do Rio de Janeiro e Região Metropolitana e ainda a grande maioria dos municípios do interior do estado do Rio de Janeiro, algumas cidades do Espírito Santo e Minas Gerais, São Paulo (capital), Distrito Federal, e outras capitais estaduais.

Por se tratar de um mercado altamente competitivo, foi realizada uma análise de custo e valor no intuito de observar fontes para reduções potenciais de custos, através de ondas de trabalho ao longo de toda a cadeia de suprimentos, onde se observou como um dos pontos focais a distribuição de produtos, mais especificamente o serviço de entrega domiciliar. $\mathrm{O}$ desenvolvimento do estudo foi acompanhado por analistas da área, em caráter observatório e em alguns momentos, participativo.

Para entender melhor este elo da cadeia de suprimentos decidiu-se avaliar internamente seus prestadores de serviços e identificar possíveis benchmarks (CAMP, 1989). Optou-se, portanto, por se utilizar a metodologia DEA, originalmente proposta por Charnes et al (1978), para analisar a eficiência das empresas distribuidoras de produtos, por se tratar de uma metodologia onde a interferência do avaliador é minimizada. Garante-se assim total transparência ao processo. Esta metodologia já é usada em determinação de bechmarks em logística e transporte. $\mathrm{Na}$ literatura destacam-se os casos estudados por Novaes (2001) e Soares de Mello et al (2003).

O presente trabalho restringe-se ao estudo de 09 empresas, localizadas na região metropolitana do Rio de Janeiro e que representam aproximadamente $70 \%$ do volume total de jornais entregues. Na seção 3 será feita uma breve descrição da metodologia DEA. As seções 4 e 5 são dedicadas ao processo de modela- gem e aplicação do modelo, respectivamente. $\mathrm{Na}$ seção 6 será realizada a interpretação dos resultados, seguida da seção 7 que traz considerações finais a respeito do estudo e sugestões para desenvolvimentos futuros da pesquisa.

\section{Metodologia DEA}

A história da Análise Envoltória de Dados começa com a dissertação para obtenção de grau de Ph.D. de Edward Rhodes sob a supervisão de W.W. Cooper publicada em 1978 (CHARNES et al 1978). O objetivo da tese foi desenvolver um modelo para estimar a eficiência técnica sem recorrer ao arbítrio de pesos para cada variável de input ou output, e sem converter todas as variáveis em valores econômicos comparáveis.

Em contraste com as aproximações paramétricas, cujo objetivo é otimizar um plano de regressão simples a partir dos dados, DEA, otimiza cada observação individual (DMU - Decision Making Unit) com o objetivo de calcular uma fronteira de eficiência. A eficiência de 100\% (DMU pertencente à fronteira) é atingida por uma unidade quando comparações com outras unidades relevantes não evidenciam ineficiência no uso de qualquer input ou output. Este conceito permite diferenciar entre estados de produção eficientes e ineficientes.

DMUs são as unidades tomadoras de decisão (decision making unit), e geralmente são representadas pelas empresas, setores ou instituições a serem avaliadas. Estas devem ser homogêneas, ou seja, atuar no mesmo tipo de negócio, realizar as mesmas tarefas com os mesmo objetivos, e estar trabalhando nas mesmas condições de mercado. As variáveis utilizadas para cada DMU devem ser as mesmas.

Há dois modelos DEA clássicos: CCR e BCC. O modelo CCR (também conhecido como CRS ou constant returns to scale), trabalha com retornos constantes de escala (CHARNES et al.,1978). Em sua formulação matemática considera-se que cada DMU $k$ $(k=1,2, \ldots, s)$ é uma unidade de produção que utiliza $n$ inputs $x i_{k}, i=1,2, \ldots, n$, para produzir $m$ outputs $y_{j k}, j=$ $1,2, \ldots, m$. Esse modelo maximiza o quociente entre a combinação linear dos outputs e a combinação linear dos inputs, com restrição de que para qualquer DMU esse quociente não seja maior que 1 .

Com alguns artifícios matemáticos, este modelo é linearizado, transformando-se no problema de programação linear (PPL) apresentado em (1), onde $h_{o}$ é a eficiência da DMU $o$ em análise; $x_{i o}$ e $y_{i o}$ são os inputs e outputs, da DMU $o ; v_{i}$ e $u_{j}$ são os pesos calculados pelo modelo para os inputs e outputs, respectivamente. 


$$
\max h_{o}=\sum_{j=1}^{m} u_{j} y_{j o}
$$

sujeito a

$\sum_{i=1}^{n} v_{i} x_{i o}=1$

$\sum_{j=1}^{m} u_{j} y_{j k}-\sum_{i=1}^{n} v_{i} x_{i k} \leq 0, \quad k=1, \ldots, s$

$u_{j}, v_{i} \geq 0 \quad \forall i, j$

O modelo BCC (BANKER et al.,1984) também chamado de VRS (variable returns to scale), considera situações de eficiência de produção com variação de escala e não assume proporcionalidade entre inputs e outputs. Por ter sido utilizado superficialmente neste estudo, o modelo BCC não será apresentado nesta seção.

Modelos com orientação ao input indicam que se deseja minimizar a utilização de recursos tal que o nível dos outputs ou produtos não se reduza. Modelos com orientação ao output indicam que o objetivo é maximizar os produtos obtidos sem alterar o nível atual dos inputs.

\subsection{Métodos de Discriminação em DEA}

\subsubsection{Fronteira Invertida}

Na fronteira invertida ou dupla envoltória (ENTANI $e t$ al., 2002), calcula-se uma fronteira de ineficiência invertendo inputs em outputs e outputs em inputs no cálculo das eficiências. A fronteira de ineficiência ajuda a identificar DMUs falso positivas, ou seja, DMUs consideradas eficientes pela fronteira padrão e ineficientes pela fronteira invertida. Para não pertencerem à fronteira invertida, as DMUs devem ser excelentes naquelas variáveis que são consideradas muito boas e não podem ser muito ruins nas outras (ANGULO MEZA et al., 2003). Um resultado conjunto das fronteiras padrão e invertida de cada DMU é obtido com a eficiência composta:

$$
\mathrm{E}_{\text {composta }}=\frac{\mathrm{E}_{\text {padrão }}+\left(1-\mathrm{E}_{\text {invertida }}\right)}{2}
$$

onde:

$\mathbf{E}_{\text {composta }}$ é a eficiência composta

$\mathbf{E}_{\text {padrão }}$ é a eficiência padrão obtida pelo cálculo da fronteira de eficiência padrão

$\mathbf{E}_{\text {invertida }}$ é a eficiência obtida pelo cálculo da fronteira de ineficiência (invertida)

\subsubsection{Restrição aos pesos}

Os pesos representam um sistema de valor relativo para cada DMU, calculados pelo PPL da equação (1), o qual fornece o melhor escore possível para cada uma, de forma que o sistema de valor resultante seja viável para todas as outras DMUs, no sentido de que nenhuma delas atinja um escore de eficiência acima de um limite superior especificado que usualmente é 1 . Na sua forma clássica, DEA permite total flexibilidade na seleção de pesos tal que cada DMU atinja o escore máximo de eficiência viável para seus níveis de inputs e outputs (LINS e ANGULO MEZA, 2000).

O interesse encontra-se em estabelecer limites entre os quais os pesos podem variar permitindo certa flexibilidade e certa incerteza sobre o verdadeiro valor dos pesos. Estes limites representam restrições adicionais na formulação original. Portanto a eficiência de uma DMU na nova formulação será menor ou igual à eficiência obtida na formulação anterior.

Existem vários enfoques para introduzir restrições nos pesos (ALCÂNTARA, et al., 2003):

\section{a) Restrições diretas nos pesos}

Nesse enfoque, desenvolvido por Dyson e Thanassoulis (1988) e generalizado por Roll e Godany (1991), são impostos limites numéricos aos multiplicadores com o objetivo de não superestimar ou ignorar inputs e outputs na análise. Os limites impostos aos multiplicadores de inputs, $\mathbf{v}_{\mathrm{i}}$, e de outputs, $\mathbf{u}_{\mathrm{i}}$, são dados pelas relações apresentadas abaixo:

$$
\begin{array}{rll}
\text { LII } & v_{i} \leq \text { LSI } \\
\text { LIO } \leq u_{j} \leq \text { LSO }
\end{array}
$$

onde:

LII é o limite inferior do input $\mathrm{i}$

LSI é o limite superior do input $\mathrm{i}$

LIO é o limite inferior do output $\mathrm{j}$

LSO é o limite superior do output j

Esse tipo de restrição pode levar à inviabilidade do PPL, já que estabelecer um limite superior ao peso de um input implica em um limite inferior no input virtual do restante das variáveis. Estellita Lins e Moreira (2001) discutem em que condições as restrições aos pesos não tornam o PPL inviável.

\section{b) Regiões de segurança}

O método de Regiões de Segurança - Assurance Region Method (AR) - desenvolvido por Thompson et al. (1990), recebe este nome pela adição de restri- 
ções aos modelos DEA clássicos que têm limites superior e inferior para cada multiplicador. Ou seja, limita a variação dos pesos a uma determinada região.

\section{b1) Região de segurança Tipo I - Método Cone Ratio}

As restrições desse enfoque, desenvolvido por Charnes et al. (1990), são exemplificadas em (5) e (6) e incorporam na análise a ordenação relativa ou valores relativos de inputs ou outputs.

$$
\begin{gathered}
\mathrm{k}_{\mathrm{i}} \mathrm{v}_{\mathrm{i}}+\mathrm{k}_{\mathrm{i}+1} \mathrm{v}_{\mathrm{i}+1} \leq \mathrm{v}_{\mathrm{i}+2} \\
\mathrm{a}_{\mathrm{i}} \leq \frac{\mathrm{v}_{\mathrm{i}}}{\mathrm{v}_{\mathrm{i}+1}} \leq \mathrm{b}_{\mathrm{i}}
\end{gathered}
$$

A formulação (6) é a mais utilizada e reflete a taxa marginal de substituição. Os valores limites são dependentes da escala das variáveis, ou seja, são sensíveis às unidades de medida. É ainda possível com o método do Cone Ratio selecionar DMUs como padrão e utilizar seus pesos como limites para o intervalo de variação dos pesos das demais DMUs.

\section{b2) Região de segurança Tipo II}

Apresentadas por Thompson et al. (1990), são restrições que relacionam os pesos de inputs e outputs, conforme (7).

$$
\gamma_{\mathrm{i}} \mathrm{v}_{\mathrm{i}} \geq \mathrm{u}_{\mathrm{j}}
$$

Em muitas aplicações de DEA são requeridas as relações entre pesos de inputs e outputs, já que a medida de eficiência reflete a combinação das variáveis. Assim como nos modelos de AR do Tipo I, os modelos do Tipo II produzem os mesmos índices de eficiência relativa, independentemente da orientação do modelo, e são igualmente influenciados pela escala das variáveis input e output.

\section{c) Restrição nos inputs e outputs virtuais}

Wong e Beasley (1990) exploraram o uso de restrições nos inputs e outputs virtuais. Por exemplo: ao invés de restringir os valores dos pesos, pode-se restringir a proporção do output virtual total da DMU i utilizado pelo output 1, ou seja, a "importância" relacionada ao output $\mathrm{r}$ pela $\mathrm{DMU} \mathrm{j}$, ao intervalo entre dois valores $\left[\Phi_{r}, \Psi_{r}\right]$, com $\Phi_{r}, \Psi_{r}$ sendo determinados pelo decisor ou usuário.

$$
\Phi_{\mathrm{r}} \leq \frac{\mathrm{u}_{1} \mathrm{y}_{1 \mathrm{k}}}{\mathrm{u}_{1} \mathrm{y}_{1 \mathrm{k}}+\mathrm{u}_{2} \mathrm{y}_{2 \mathrm{k}+\ldots+} \mathrm{u}_{\mathrm{n}} \mathrm{y}_{\mathrm{nk}}} \leq \Psi_{\mathrm{r}}
$$

onde:

k é a DMU que está sendo avaliada

y são os valores dos outputs

n é o número total de outputs

O mesmo raciocínio pode ser aplicado para se restringir a proporção dos inputs.

\section{Modelagem}

O modelo utilizado visa analisar a eficiência das empresas, tendo em vista tanto os aspectos operacionais quanto de qualidade da entrega realizada.

Indica-se que o número de DMUs deve ser o triplo (no mínimo) do número de variáveis utilizadas no modelo, em se tratando de modelos DEA tradicionais. Estudos mais recentes (GONZALEZ ARAYA, 2003) sugerem que essa relação deve ser de 4 ou 5 para um.

A introdução de um grande número de variáveis resulta em uma maior explicação causal do modelo, mas por outro lado, fará com que um número maior de DMUs esteja na fronteira, ou seja, o incremento de muitas variáveis reduz a capacidade de DEA de discriminar as DMUs eficientes das ineficientes. Portanto, o modelo deve ser mantido o mais compacto possível para maximizar o poder discriminatório de DEA(LINS e ANGULO MEZA, 2000).

As DMUs são representadas pelas empresas distribuidoras, pertencentes ao escopo do trabalho, totalizando 9 empresas. Para a avaliação, foram usadas três variáveis, sendo 2 inputs e 1 output, a saber:

Input 1: Número de entregadores

A Infoglobo possui preocupação especial com o número de entregadores em sua operação, que é realizada durante a madrugada e início da manhã. Quanto menor o número de entregadores, supõe-se maior eficiência da empresa em gerenciar sua mão-de-obra, assim como otimizar roteiros e horários de entrega, convertidos assim em menores custos. Atentamos para o fato desta variável não contemplar o número de supervisores e gerentes envolvidos na operação.

\section{Input 2: Número de Reclamações}

Refere-se ao número total de reclamações recebidas no período de um mês, através da Central de Atendimento da Infoglobo. Estas reclamações contemplam produtos não entregues, atrasos na entrega e recebimento do produto em condições inadequadas. Esta variável impacta diretamente no faturamento 
das empresas terceirizadas, devido ao sistema de metas em vigor, que visa melhorar a qualidade da entrega premiando ou punindo as empresas pelo seu desempenho na operação.

Na verdade, esta variável é um output indesejável (TAVARES, 1998), pois quanto menor for o seu valor, supõe-se que melhor será o cálculo da eficiência da empresa. Entretanto, para o cálculo coerente das eficiências, esta variável deve constar no modelo como um falso input.

Output 1: Número de Jornais entregues

Representa o volume de jornais entregues mensalmente pelas empresas. Associado ao número de reclamações, compõe o resultado da operação.

Os dados utilizados referem-se ao mês de agosto de 2003. Os valores utilizados e a identificação das empresas não serão divulgados, sendo as empresas representadas por letras.

\section{Aplicação}

Optou-se por utilizar o modelo CRS, por se tratar de uma avaliação menos benevolente e, portanto com maior caráter discriminatório que o modelo VRS. A escolha do modelo é uma interferência do analista, e preferências do decisor podem ser modeladas através de restrições aos pesos (ver seção 5.2). O modelo foi orientado a input, pois se deseja minimizar os recursos utilizados na operação tal que o nível de outputs não se reduza.

Apesar da diferença na escala de operação das empresas (DMUs), as variáveis utilizadas são iguais mudando apenas sua magnitude em função da escala de operação, porém seguindo uma proporcionalidade visto que na variável entregadores não foi contemplado o número de funcionários envolvidos na supervisão e coordenação das atividades, desta forma pressupõese que os entregadores possuam igual grau de capacidade de entrega independente da DMU considerada, permitindo assim a utilização do modelo CRS.

Para o cálculo das eficiências, utilizou-se o software SIAD (ANGULO MEZA et al., 2003), e os resultados são apresentados a seguir:

Tabela 1 - Eficiências - Modelo CRS orientado a Input.

\begin{tabular}{cc}
\hline DMUs & $\begin{array}{c}\text { Eficiência CRS - sem } \\
\text { restrições aos pesos }\end{array}$ \\
\hline DMU A & 1,00 \\
\hline DMU B & 0,89 \\
\hline DMU C & 0,63 \\
\hline DMU D & 0,70 \\
\hline DMU E & 0,64 \\
\hline DMU F & 0,46 \\
\hline DMU G & 0,49 \\
\hline DMU H & 0,49 \\
\hline DMU I & 0,38
\end{tabular}

Em DEA, muitas vezes é difícil visualizar a fronteira de eficiência, em função do número de variáveis utilizados no modelo. No caso deste estudo, através das relações Output/Input 1 e Output/Input 2, pode-se ilustrar a fronteira CRS obtida, sem restrições aos pesos.

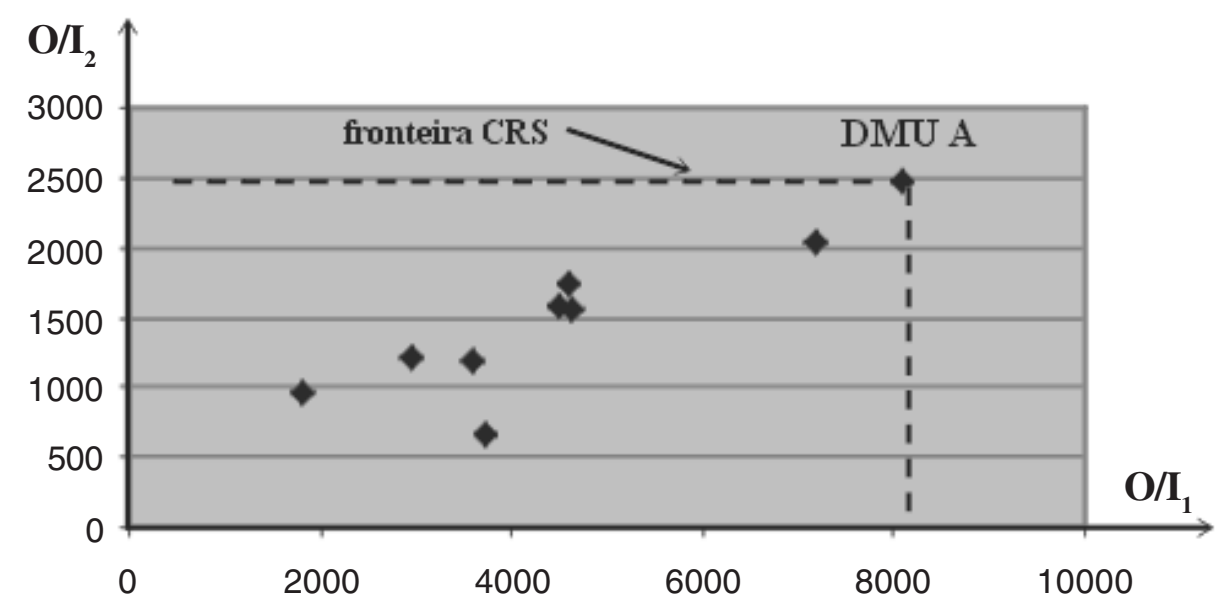

Figura 1. Fronteira CRS: O/I $\mathrm{X} \mathrm{O}_{1} \mathrm{I}_{2}$ 
Observa-se no gráfico que a DMU A é eficiente (valor igual a 1,00) e assim, pertence à fronteira. A DMU B $(0,89)$ ficou próxima à fronteira de eficiência, o que mostra um bom desempenho desta unidade. Todas as outras DMUs tiveram suas eficiências iguais ou abaixo de 0,70, o que leva a crer que as DMUs A e B realmente se destacam na atividade, sem enfrentar grande competitividade. Esta disparidade levou o modelo a uma eficiência média de 0,63 , relativamente baixa, o que representa um baixo nível de eficiência operacional na atividade.

\subsection{Uso do Modelo VRS}

Também foi testado o modelo VRS, orientado a input, para obter as eficiências das empresas. Obtiveram-se duas DMUs eficientes: a DMU A, exatamente como no modelo CRS, e a DMU F, que mostrou-se eficiente por default. Utilizando os recursos da fronteira invertida e da eficiência composta (ver seção 3.1.1), para uma melhor discriminação dos resultados, a eficiência da DMU F obtida no modelo VRS revelouse uma falsa eficiência, já que na análise da fronteira invertida o resultado obtido foi 1,00, ou seja, a pior eficiência. A ordenação final dos resultados pela eficiência composta foi bastante similar à encontrada no modelo CRS, mas devido à falsa eficiência apresentada decidiu-se por não utilizar este modelo.

\subsection{Formulação de Restrições}

Ao se analisar os resultados obtidos pelo modelo CRS, percebeu-se que a importância da variável Número de Entregadores na formação da eficiência das DMUs foi muito pequena, o que foi evidenciado pelos pesos obtidos nesta variável: das 9 DMUs avaliadas, 7 delas obtiveram peso zero. Ao discutir os resultados obtidos com analistas da Infoglobo, decidiu-se que esta situação deveria ser evitada, já que desprezava o aspecto operacional da atividade de entrega domiciliar.

Para valorizar o aspecto operacional, aplicou-se uma restrição ao input virtual a todas as DMUs, já que neste método pode-se estabelecer a importância relativa de variáveis na formação da eficiência final. Desta forma, estabeleceu-se limites para que a importância relativa do input Número de Entregadores assumisse valores entre $50 \%$ e $75 \%$, valores estes definidos em conjunto com analistas da Infoglobo, por meio de suas experiências e expectativas sobre a avaliação.

$$
\begin{gathered}
0.5 \leq \frac{\mathrm{X}_{1 \mathrm{i}} \mathrm{n}_{1 \mathrm{i}}}{\mathrm{X}_{1 \mathrm{i}} \mathrm{v}_{1 \mathrm{i}}+\mathrm{X}_{2 \mathrm{i}} \mathrm{v}_{2 \mathrm{i}}} \leq 0.75 \\
\text { onde } \mathrm{i}=1,2,3 \ldots . .9 \text { (DMUs) }
\end{gathered}
$$

A seguir, são apresentados os novos resultados obtidos com as restrições aplicadas, calculadas pelo software LINDO, uma vez que o software SIAD, até o fechamento deste artigo, não possuía a funcionalidade de restrição ao input e output virtual.

Tabela 2 - Comparação de Eficiências - Modelo CRS

\begin{tabular}{lcc}
\hline DMUs & $\begin{array}{c}\text { Eficiência CRS - sem } \\
\text { restrições aos pesos }\end{array}$ & $\begin{array}{c}\text { Eficiência CRS - com } \\
\text { restrições aos pesos }\end{array}$ \\
\hline DMU A & 1,00 & 1,00 \\
\hline DMU B & 0,89 & 0,86 \\
\hline DMU C & 0,63 & 0,61 \\
\hline DMU D & 0,70 & 0,59 \\
\hline DMU E & 0,64 & 0,59 \\
\hline DMU F & 0,46 & 0,46 \\
\hline DMU G & 0,49 & 0,40 \\
\hline DMU H & 0,49 & 0,36 \\
\hline DMU I & 0,38 & 0,27 \\
\hline
\end{tabular}

Nota-se que as eficiências calculadas com restrição aos pesos da variável "Número de Entregadores" apresentam valores inferiores às calculadas sem restrição, como era de se esperar (ver seção 3.1.2). Como consequiência, a eficiência média do modelo com restrição foi reduzida para 0,57 .

Tabela 3 - Comparação da Ordenação das DMUs - Modelo CRS

\begin{tabular}{ccc}
\hline Ordenação & $\begin{array}{c}\text { Eficiência CRS - sem } \\
\text { restrições aos pesos }\end{array}$ & $\begin{array}{c}\text { Eficiência CRS - com } \\
\text { restrições aos pesos }\end{array}$ \\
\hline 1 & DMU A & DMU A \\
\hline 2 & DMU B & DMU B \\
\hline 3 & DMU D & DMU C \\
\hline 4 & DMU E & DMU D \\
\hline 5 & DMU C & DMU E \\
\hline 6 & DMU G & DMU F \\
\hline 7 & DMU H & DMU G \\
\hline 8 & DMU F & DMU H \\
\hline 9 & DMU I & DMU I
\end{tabular}

A partir da restrição aplicada ao modelo, mudanças consideráveis ocorreram na ordenação das DMUs segundo as eficiências, principalmente nas posições intermediárias, conferindo uma melhor discriminação e validação do modelo, que reflete a sensibilidade dos analistas da Infoglobo em relação à operação, e que justifica a implementação das restrições.

\section{Resultados}

Para auxiliar na interpretação dos resultados, apresentase a seguir a área geográfica de entrega de cada empresa, e também introduz-se a variável índice de verticalização. 
Tabela 4 - Áreas de Entrega e Índice de Verticalização

\begin{tabular}{cclc}
\hline Ordenação & DMUs & \multicolumn{1}{c}{ Local de Entrega } & Índice de Verticalização \\
\hline 1 & DMU A & IPANEMA, LEBLON, COPACABANA & $93,6 \%$ \\
\hline 2 & DMU B & BOTAFOGO, FLAMENGO & $92,3 \%$ \\
\hline 3 & DMU C & TIJUCA, MARACANÃ & $84,3 \%$ \\
\hline 4 & DMU D & NITEROI (ICARAÍ), SÃO GONÇALO & $65,0 \%$ \\
\hline 5 & DMU E & RECREIO, BARRA & $79,2 \%$ \\
\hline 6 & DMU F & VILA ISABEL & $63,4 \%$ \\
\hline 7 & DMU G & ZONA OESTE & $34,7 \%$ \\
\hline 8 & DMU H & NITEROI (REGIAO OCEÂNICA) & $29,9 \%$ \\
\hline 9 & DMU I & BAIXADA FLUMINENSE & $31,7 \%$ \\
\hline
\end{tabular}

Como se pode perceber, as empresas mais eficientes (A, B e C) estão localizadas em áreas com índices de verticalização ${ }^{1}$ elevados e com menor grau de dispersão dos pontos de entrega.

Ao observarmos as DMUs com eficiências mais baixas (G, H e I), suas áreas de atuação são altamente dispersas e com baixo índice de verticalização em sua maior parte.

A partir desta análise, abre-se espaço para uma discussão a respeito da inclusão de novas variáveis para contemplar algumas diferenças como, no exemplo deste artigo, as características das áreas geográficas de atuação das empresas. A inclusão de novas variáveis pode tornar o modelo mais exaustivo; no entanto, é preciso observar o que se pretende medir e a relação entre o número de variáveis e a quantidade de DMUs. A inclusão de variáveis em excesso pode tornar as DMUs todas eficientes, o que inviabiliza qualquer análise. Assim, as variáveis que ficam fora do modelo podem ser consideradas variáveis explicativas das ineficiências. Devemos estar atentos que, em DEA, quanto maior o número de variáveis, menor a capacidade discriminatória do modelo. Para se manter uma boa discriminação, recomenda-se uma relação de 5: 1 entre DMUs e variáveis (inputs e outputs) (ver seção 4).

\subsection{Simulação de Fusão de Empresas}

Observando as DMUs D e H, ambas atuando na região de Niterói e com eficiências relativamente baixas, aplicou-se uma simples técnica de fusão de empresas em DEA, através da soma dos inputs e outputs (AVELLAR et al., 2002). A seguir, recalculou-se a eficiência de todas as DMUs, incluindo a nova empresa, proveniente da fusão. Na tabela 4, pode-se observar o resultado obtido. Para auxiliar na interpretação dos resultados, a DMU D possui cerca $80 \%$ dos jornais entregues na região, ficando a DMU H com os $20 \%$ restantes.

Tabela 5 - Comparação de Eficiências após a fusão

\begin{tabular}{cc}
\hline DMUs & $\begin{array}{c}\text { Eficiência - CRS com } \\
\text { restrição aos pesos }\end{array}$ \\
\hline DMU D & 0,59 \\
\hline DMU H & 0,36 \\
\hline FUSÃO* & 0,53 \\
\hline
\end{tabular}

A eficiência da nova empresa é um valor intermediário entre as eficiências das empresas anteriores à fusão.

Para a empresa D, a fusão pode representar uma péssima estratégia, já que terá sua eficiência reduzida em aproximadamente $6 \%$, em troca de um pequeno acréscimo no volume de entregas e conseqüentemente pequeno aumento em seu faturamento.

Para a empresa $\mathrm{H}$, pode representar uma boa tentativa de alavancagem do negócio, pois apresenta um ganho de eficiência de aproximadamente 17\%, além de um grande aumento no volume de entregas, o que impacta diretamente o faturamento da empresa. Além disso, a eficiência média do modelo, com a inclusão da DMU FUSÃO*, passa a ser de 0,59 , contra 0,57 do modelo anterior, contendo as duas empresas.

Desta forma, conclui-se que a fusão aditiva destas empresas, neste caso específico, não é uma boa

1 Índice de verticalização $=1-($ no pontos de entrega / no de produtos entregues)

Exemplo: $I_{v}=0,9$ pode representar uma densa área residencial onde os jornais são entregues na portaria dos edifícios, ou seja, um número maior de jornais por ponto de entrega. De forma análoga, $I_{v}=0,1$ pode representar uma área residencial onde a maioria dos pontos de entrega são casas (poucos jornais por ponto de entrega). 
alternativa para a Infoglobo, pois a fusão não representaria um ganho mínimo de eficiência para o serviço com um todo, e um grande decréscimo de eficiência na DMU D, onde a maior parte das entregas da região (cerca de 80\%) está concentrada.

\section{Comentários finais}

A abordagem DEA oferece uma contribuição significativa neste estudo específico por fornecer uma modelagem com mínima ou pouca interferência do avaliador, importantes em processos de avaliação, principalmente de empresas prestadoras de serviços, e por permitir a simulação de empresas fictícias (exemplo:fusão de empresas) comparando previamente o efeito de sua inclusão nos resultados globais.

Outra contribuição relevante trata-se da definição de empresas benchmarks, a partir da qual pode ser realizado um trabalho de mapeamento de processos para a definição de padrões de operação e metas de desempenho. Este processo se tornaria altamente dispendioso e até mesmo inviável caso fosse realizado um estudo em todas as empresas (DMUs) e posterior análise de especialistas, o que certamente desconsideraria aspectos de eficiência da operação, devido à complexidade do seu cálculo de forma objetiva e mensurável através de outras técnicas e abordagens, por se tratar de uma atividade de múltiplos inputs e outputs.

Ratifica-se, ainda, a importância prática da aplicação do modelo construído, observado por analistas da Infoglobo que acompanharam o estudo desde o início e garantiram a sustentabilidade dos resultados obtidos.

Para futuros estudos, sugere-se a abertura das empresas em suas respectivas áreas de entrega, e sua divisão em clusters, segundo os índices de verticalização e grau de dispersão de entrega de cada área. Em seguida, estas áreas podem ser avaliadas dentro de seu respectivo cluster, e ordenadas segundo suas eficiências, observando-se as melhores práticas (benchmarks).

\section{Referências}

ADLER, N.; GOLANY, B. Evaluation of deregulated airline networks using data envelopment analysis combined with principal component analysis with an application to Western Europe. European Journal of Operational Research, v. 132, n. 2, 2001, p. 260-273.

ALCÂNTARA, A.A.; SANT'ANNA, A.P.; LINS, M.P.E. Restringindo flexibilidade de pesos em DEA utilizando análise de regressão MSEA. 2003. nação de benchmarks de companhia aérea brasileiras DEA - ineficientes. Anais do XVI ANPET, 27-34, 2002.

ANGULO MEZA, L.; BIONDI NETO, L.; SOARES DE MELLO, J.C.C.B.; GOMES, E.G. SIAD - Sistema Integrado de Apoio à Decisão: uma implementação computacional de modelos de Análise Envoltória de Dados. Resumos da I Reunião Regional da Sociedade Brasileira de Pesquisa Operacional no Rio de Janeiro, Junho, Niterói, RJ, 24-24, 2003.

AVELLAR, J.V.G.; POLEZZI. A.O.D.; MILIONI, A.Z. On the evalution of Brazilian landline telephone services companies. Revista Pesquisa Operacional, 22, no.2, Jul/Dez 2002.

BANKER, R.D.; CHARNES, A.; COOPER, W.W. Some models for estimating technical scale inefficiencies in Data Envelopment Analysis. Management Science, v.30, n.9, 1984, p. 1078-1092.

CAMP, C. Benchmarking: the search for best practices wich lead for superior performance. Quality Progress, New York, 1989.

CHARNES, A.; COOPER, W. W.; HUANG, Z. M.; SUN, D. B. (1990). Polyhedral Cone-Ratio DEA Models with an illustrative application to Large Commercial Banks. Journal of Econometrics, $\mathrm{n}^{\circ} 46$, pp. 73-91.

CHARNES, A.; COOPER, W.W.; RHODES, E. Measuring the efficiency of decision-making units. European Journal of Operational Research, v.2, 1978, p. 429-444.

DYSON, R. G.; THANASSOULIS E. (1988) Reducing weight flexibility in DEA. Journal of the Operational Research Society, 39.

ENTANI, T.; MAEA, Y.; TANAKA, H. (2002) Dual Models of Interval DEA and its extensions to interval data. European Journal of Operational Research, 136, 32-45.

ESTELLITA LINS, M. P.; MOREIRA, A. C. (2001) Evitando a Inviabilidade em Modelos DEA com Restrições aos Pesos. Relatório Técnico EP-03/01, Programa de Engenharia de Produção/COPPE/UFRJ, Rio de Janeiro, RJ, Brasil.

GOMES, E.G.; SOARES DE MELLO, J.C.C.B.; SERAPIÃO, B.P.; LINS, M.P.E.; BIONDI NETO, L. Avaliação de Eficiência de Companhias Aéreas Brasileiras: Uma abordagem por Análise Envoltória de Dados. In: Setti, J.R.A. e O.F. Lima Junior (eds), 
Nanci, L. C.; Azeredo, S. M.; Mello, J. C. C. B. S. - Estudo da eficiência de empresas distribuidoras de jornais...

Panorama Nacional da Pesquisa em Transportes 2001 - Anais do XV ANPET, Campinas, SP, Novembro, v.2, 2001, p.125-133.

GONZÁLEZ-ARAYA, M.C. Projeções Não Radiais em Regiões Fortemente Eficientes da Fronteira DEA - Algoritmos e Aplicações. Tese de Doutorado, Programa de Engenharia de Produção, COPPE/UFRJ, Rio de Janeiro. 2003.

LINS, M.P.E.; ANGULO MEZA, L. Análise Envoltória de Dados. COPPE/UFRJ, Rio de Janeiro, 2000.

NOVAES, A.G. Logística e Gerenciamento da Cadeia de Distribuição. Ed. Campus. Rio de Janeiro, 2001.

NOVAES, A.G. Rapid-transit efficiency analysis with the Assurance-Region DEA method. Pesquisa Operacional, v.21, n.2, 2001, p.179-197.

ROLL, Y.; GOLANY, B. (1991) Controlling factor weights in DEA. IIE Transactions, 23 (1), pp.2-9.
SOARES DE MELLO, J.C.C.B.; ANGULO MEZA, L.; GOMES, E.G.; SERAPIÃO, B.; LINS, M.P.E. Análise envoltória de dados no estudo da eficiência e dos benchmarks para companhia aéreas brasileiras. Pesquisa Operacional, 23, 2003, 325-345.

TAVARES, G. DEA - “Data Envelopment Analysis": Os modelos básicos e as suas extensões principais Um Modelo para a Análise da Modernização dos Serviços de Telecomunicações nos Países da OCDE. Tese de mestrado, Departamento de Engenharia Informática, Universidade de Coimbra, Coimbra. 1998.

THOMPSON, R. G.; LANGEMEIER, L. N.; LEE, C. H.; LEE, E.; THRALL, R. M. (1990) The role of multiplier bounds in efficiency analysis with application to Kansas farming. Journal of Econometrics, 46, pp. 93-108.

WONG, Y.; BEASLEY, J. "Restricting Weight Flexibility in DEA". Journal of Operational Research Society, 41, 1990, 829-835.

Submetido em junho/2004

Aprovado em outubro/2004 